\title{
Methods for model error analysis of pulse impulse measuring torsion system
}

\author{
Xing Jin ${ }^{a}$, Dapeng Wang ${ }^{b^{*}}$ and Chao Zhu ${ }^{\mathrm{c}}$ \\ State Key Laboratory of Laser Propulsion \& Application, Equipment Academy, Beijing 101416, \\ China \\ ajinxing_beijing@sina.com, bajwzajwz@163.com, 'Zhuchaod1630@163.com
}

Keywords: Torsion system, impulse measurement, error analysis

Abstract. Torsion system is a typical pulse impulse measuring method. Currently used analysis model is the impulse moment interaction model. According to the response characteristic analysis of torsion system under pulsed impulse action, using analytical methods, this thesis proposes error analysis methods for impulse momentary action model and also proposes the main factors of model error is damping ratio of torsion system, the ratio of pulse time as well as system impulse period. The proposed model error analysis methods facilitate pulse impulse error analysis, which provides analysis and design methods for impulse pulse measurement and error analysis in engineering.

\section{Introduction}

In engineering, the thrust is considered as impulse force when action time is short, then we measure impulse that thrust generated. For example, when action time is less than $1 / 4$ of torsion vibration period, changes of thrust over time is difficult to identify due to the limited number of sampling points of system response. Then effects of thrust perform as impulse effect and thrust measurement is converted into impulse measurement ${ }^{[1,2]}$.

The current used model is momentary impulse action model, but the actual thrust always has action time, so there is a certain discrepancy between impulse measurement results and the actual situation, and it is needed to determine methods of analyzing model errors and the process of how model errors act so as to increase impulse measurement accuracy ${ }^{[3,4]}$.

\section{Momentary impulse role model}

Under moment impulse action, the torsion system response is shown below.

$$
\theta(t)=\frac{S L_{f}}{J \omega_{d}} e^{-\zeta \omega_{n} t} \sin \omega_{d} t
$$

where, $L_{f}$ is the arm; $\zeta$ is damping ratio; $\omega_{d}$ is the torsion vibration frequency $\left(\omega_{d}=\sqrt{1-\zeta^{2}} \omega_{n}\right) ; J$ is the moment of inertia.

The relationship of torsion angle $\theta\left(t_{M k}\right)$ and time $t_{M k}$ of system response curve's extreme points and impulse measured is shown in Eq. 2.

$$
S_{k}=\frac{J \omega_{d} e^{\zeta \omega_{n} t_{M k}}}{L_{f} \sin \omega_{d} t_{M k}} \theta\left(t_{M k}\right) \quad(k=1,2, \mathbf{L})
$$

If the j-th impulse measurement is $S_{j}$, the sample mean and sample standard deviation of the impulse is shown in Eq. 3.

$$
\bar{S}=\frac{1}{n} \sum_{j=1}^{n} S_{j}, S_{S}=\sqrt{\frac{1}{n-1} \sum_{j=1}^{n}\left(S_{j}-\bar{S}\right)^{2}}
$$


where, $n$ is the number of measurements, and it can be used to estimate the confidence interval.In the above, the thrust time is negligible and the system response is the effect of the momentary impulse, so impulse measurement and calculation methods are called impulse momentary action models ${ }^{[5]}$.

\section{Error analysis methods of momentary impulse action model}

Momentary impulse action model is an ideal model that regards the action time as infinitesimal (negligible), while the actual thrust always has action time. The below will research on errors of the momentary impulse action model.

Set the thrust as $f(t)=f_{0}\left(0 \leq t \leq T_{0}\right)$, the action time of thrust as $T_{0}$, the arm as $L_{f}$, the moment as $M(t)=f_{0} L_{f}$, then the torsion system response is shown as below.

$$
\begin{aligned}
\theta_{1}(t) & =\frac{L_{f}}{J \omega_{d}} \int_{0}^{t} f(\tau) e^{-\zeta \omega_{n}(t-\tau)} \sin \omega_{d}(t-\tau) \mathrm{d} \tau \\
& =\frac{f_{0} T_{0} L_{f}}{J \omega_{d} T_{0}} \int_{0}^{t} e^{-\zeta \omega_{n}(t-\tau)} \sin \omega_{d}(t-\tau) \mathrm{d} \tau \\
& =\frac{S L_{f}}{J \omega_{d}} \cdot \frac{1}{T_{0}} \int_{0}^{t} e^{-\zeta \omega_{n}(t-\tau)} \sin \omega_{d}(t-\tau) \mathrm{d} \tau
\end{aligned}
$$

Where, $S=f_{0} T_{0}$ is relevant impulse of the thrust. When $t>T_{0}$, set initial torsion angle as $\theta_{1}\left(T_{0}\right)$, the initial angular velocity as $\theta_{1}^{\&}\left(T_{0}\right)$, then the system begin to vibrate freely, and the below is get.

$$
\begin{gathered}
\theta_{1}\left(T_{0}\right)=\frac{S L_{f}}{J \omega_{d}} \cdot \frac{1}{T_{0}} \int_{0}^{T_{0}} e^{-\zeta \omega_{n}\left(T_{0}-\tau\right)} \sin \omega_{d}\left(T_{0}-\tau\right) \mathrm{d} \tau \\
\theta_{1}^{\&}\left(T_{0}\right)=\frac{S L_{f} \omega_{n}}{J \omega_{d}} \cdot \frac{1}{T_{0}} \int_{0}^{T_{0}} e^{-\zeta \omega_{n}\left(T_{0}-\tau\right)} \cos \left[\omega_{d}\left(T_{0}-\tau\right)+\alpha_{1}\right] \mathrm{d} \tau \\
\alpha_{1}=\arctan \frac{\zeta \omega_{n}}{\omega_{d}}=\arctan \frac{\zeta}{\sqrt{1-\zeta^{2}}}
\end{gathered}
$$

When $t>T_{0}$, the system response of free vibration stage is shown in Eq.5 below.

$$
\begin{array}{r}
\theta_{2}\left(t-T_{0}\right)=\sqrt{\theta_{1}^{2}\left(T_{0}\right)+\left[\frac{\theta_{1}\left(T_{0}\right) \zeta \omega_{n}+\theta_{1}^{\delta}\left(T_{0}\right)}{\omega_{d}}\right]^{2}} e^{-\zeta \omega_{n}\left(t-T_{0}\right)} \sin \left[\omega_{d}\left(t-T_{0}\right)+\alpha_{2}\right] \\
\alpha_{2}=\arctan \frac{\theta_{1}\left(T_{0}\right) \omega_{d}}{\theta_{1}\left(T_{0}\right) \zeta \omega_{n}+\theta_{1}^{\delta}\left(T_{0}\right)}
\end{array}
$$

For ease of discussion, dimensionless quantity is introduced.

$$
\begin{gathered}
A=\frac{1}{T_{0}} \int_{0}^{T_{0}} e^{-\zeta \omega_{n}\left(T_{0}-\tau\right)} \sin \omega_{d}\left(T_{0}-\tau\right) \mathrm{d} \tau \\
B=\frac{1}{T_{0}} \int_{0}^{T_{0}} e^{-\zeta \omega_{n}\left(T_{0}-\tau\right)} \cos \left[\omega_{d}\left(T_{0}-\tau\right)+\alpha_{1}\right] \mathrm{d} \tau
\end{gathered}
$$

Then the below can be get.

$$
\begin{gathered}
\theta_{1}\left(T_{0}\right)=\frac{S L_{f}}{J \omega_{d}} \cdot \frac{1}{T_{0}} \int_{0}^{T_{0}} e^{-\zeta \omega_{n}\left(T_{0}-\tau\right)} \sin \omega_{d}\left(T_{0}-\tau\right) \mathrm{d} \tau=\frac{S L_{f} A}{J \omega_{d}} \\
\frac{\theta_{1}\left(T_{0}\right) \zeta \omega_{n}}{\omega_{d}}=\frac{S L_{f} \zeta \omega_{n}}{J \omega_{d}^{2}} \cdot \frac{1}{T_{0}} \int_{0}^{T_{0}} e^{-\zeta \omega_{n}\left(T_{0}-\tau\right)} \sin \omega_{d}\left(T_{0}-\tau\right) \mathrm{d} \tau=\frac{S L_{f} \zeta \omega_{n} A}{J \omega_{d}^{2}} \\
\frac{\theta_{1}^{\delta\left(T_{0}\right)}}{\omega_{d}}=\frac{S L_{f} \omega_{n}}{J \omega_{d}^{2}} \cdot \frac{1}{T_{0}} \int_{0}^{T_{0}} e^{-\zeta \omega_{n}\left(T_{0}-\tau\right)} \cos \left[\omega_{d}\left(T_{0}-\tau\right)+\alpha_{1}\right] \mathrm{d} \tau=\frac{S L_{f} \omega_{n} B}{J \omega_{d}^{2}}
\end{gathered}
$$




$$
\begin{gathered}
\sqrt{\theta_{1}^{2}\left(T_{0}\right)+\left[\frac{\theta_{1}\left(T_{0}\right) \zeta \omega_{n}+\theta_{1}^{\delta}\left(T_{0}\right)}{\omega_{d}}\right]^{2}}=\frac{S L_{f}}{J \omega_{d}} \sqrt{A^{2}+\left(\frac{\zeta A+B}{\sqrt{1-\zeta^{2}}}\right)^{2}} \\
\alpha_{2}=\arctan \frac{\theta_{1}\left(T_{0}\right) \omega_{d}}{\theta_{1}\left(T_{0}\right) \zeta \omega_{n}+\theta_{1}^{\&(}\left(T_{0}\right)}=\arctan \frac{\sqrt{1-\zeta^{2}} A}{\zeta A+B}
\end{gathered}
$$

Thus, the free vibration Equation is rewritten as below.

$$
\theta_{2}\left(t-T_{0}\right)=\frac{S L_{f}}{J \omega_{d}} \sqrt{A^{2}+\left(\frac{\zeta A+B}{\sqrt{1-\zeta^{2}}}\right)^{2}} e^{-\zeta \omega_{n}\left(t-T_{0}\right)} \sin \left[\omega_{d}\left(t-T_{0}\right)+\alpha_{2}\right]
$$

As described above, on conditions of impulse $S$ and action time $T_{0}$, the system response Equation is different from momentary impulse action model.

The time and torsion angles corresponding to the extreme points of system response curves are given respectively as below.

$$
\begin{gathered}
\omega_{d}\left(t_{M k}-T_{0}\right)+\alpha_{2}=\frac{(2 k-1) \pi}{2} \quad(k=1,2, \mathrm{~L}) \\
\theta_{2}\left(t_{M k}-T_{0}\right)=\frac{S L_{f}}{J \omega_{d}} \sqrt{A^{2}+\left(\frac{\zeta A+B}{\sqrt{1-\zeta^{2}}}\right)^{2}} e^{-\frac{\zeta}{\sqrt{1-\zeta^{2}}}\left[\frac{(2 k-1) \pi}{2}-\alpha_{2}\right]} \quad(k=1,2, \mathrm{~L})
\end{gathered}
$$

Take $t_{M k}$ and $\theta_{2}\left(t_{M k}-T_{0}\right)$ ( equivalent to actual measurement results ) into momentary impulse action model, then the value of estimated impulse $S^{\prime}$ can be expressed as below.

$$
\frac{S L_{f}}{J \omega_{d}} \sqrt{A^{2}+\left(\frac{\zeta A+B}{\sqrt{1-\zeta^{2}}}\right)^{2}} e^{-\frac{\zeta}{\sqrt{1-\zeta^{2}}}\left[\frac{(2 k-1) \pi}{2}-\alpha_{2}\right]}=\frac{S^{\prime} L_{f}}{J \omega_{d}} e^{-\zeta \omega_{n} t_{M k}}\left|\sin \omega_{d} t_{M k}\right|
$$

The actual acted impulse is $S$. According to momentary impulse action model, the estimated impulse is $S^{\prime}$, and then the relative error of measuring impulse is given as below.

$$
\varepsilon_{S}=\frac{S^{\prime}-S}{S}=\frac{\sqrt{A^{2}+\left(\frac{\zeta A+B}{\sqrt{1-\zeta^{2}}}\right)^{2}} e^{-\frac{\zeta}{\sqrt{1-\zeta^{2}}}\left[\frac{(2 k-1) \pi}{2}-\alpha_{2}\right]}}{e^{-\frac{\zeta}{\sqrt{1-\zeta^{2}}}\left[\omega_{d} T_{0}+\frac{(2 k-1) \pi}{2}-\alpha_{2}\right]} \mid \sin \left[\omega_{d} T_{0}+\frac{(2 k-1) \pi}{2}-\alpha_{2}\right]}-1
$$

By combination of the above analysis, the relative error formula of momentary impulse action model is given as Eq. 6.

$$
\varepsilon_{S}=\frac{S^{\prime}-S}{S}=\frac{\sqrt{A^{2}+\left(\frac{\zeta A+B}{\sqrt{1-\zeta^{2}}}\right)^{2}} \frac{\zeta}{e^{\sqrt{1-\zeta^{2}}} \omega_{d} T_{0}}}{\left|\sin \left[\omega_{d} T_{0}+\frac{(2 k-1) \pi}{2}-\alpha_{2}\right]\right|}-1
$$

where, $k=1,2$, $\mathrm{r}$ respectively corresponds to the first maximum value, the first minimum value, the second maximum value and the second minimum value, etc. , so is the after.

The action time of is expressed as multiple of system cycles $T=2 \pi / \omega_{d}: T_{0}=k_{T} T=k_{T}\left(2 \pi / \omega_{d}\right)$, then the calculation formula of model errors is given as below.

$$
\varepsilon_{S}=\frac{S^{\prime}-S}{S}=\frac{\sqrt{A^{2}+\left(\frac{\zeta A+B}{\sqrt{1-\zeta^{2}}}\right)^{2}} e^{\frac{\zeta}{\sqrt{1-\zeta^{2}}}\left(2 \pi k_{r}\right)}}{\left|\sin \left[2 \pi k_{T}+\frac{(2 k-1) \pi}{2}-\alpha_{2}\right]\right|}-1=\frac{\sqrt{A^{2}+\left(\frac{\zeta A+B}{\sqrt{1-\zeta^{2}}}\right)^{2}} \frac{\zeta}{e^{\sqrt{1-\zeta^{2}}}\left(2 \pi k_{T}\right)}}{\left|\sin \left[2 \pi k_{T}-\frac{\pi}{2}-\alpha_{2}\right]\right|}-1
$$


Obviously, the main error factors of momentary impulse action model is damping ratio and cycle multiples $k_{T}$, and independent of other factors.

\section{Analysis and discussion}

Fig. 1 and Fig. 2 shows relative errors of momentary impulse action model changes with variation of torsion damping ratio and cycle multiples.

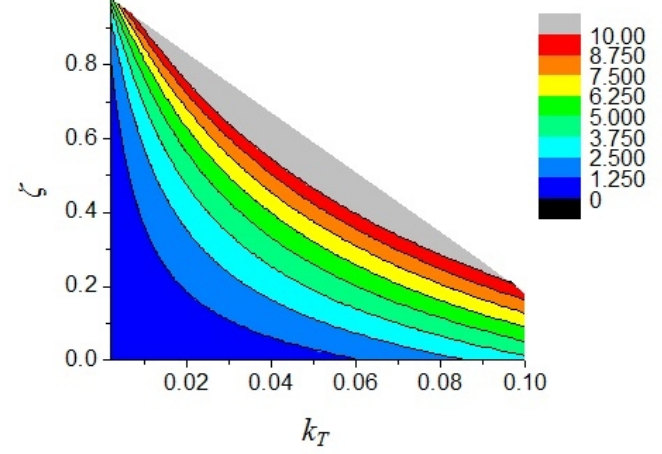

Fig. 1 relative errors(\%)changes with $k_{T}$ and $\zeta$

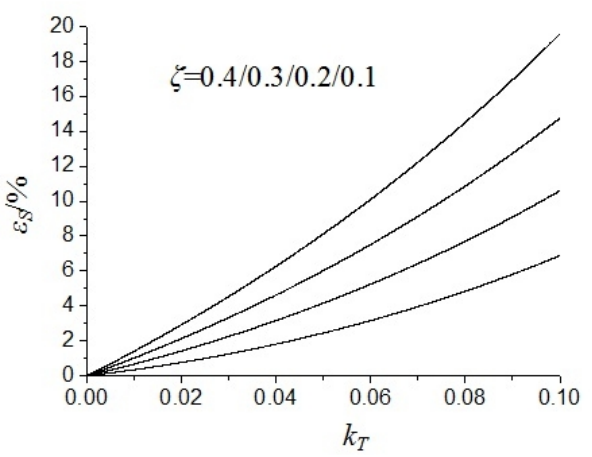

Fig. 2 relative errors changes with $k_{T}$

Figure 1 shows the relative error $\varepsilon_{S}$ (expressed in \%) increases with increasing of $k_{T}$ and $\zeta$. The calculation results show when $k_{T}=0.1$, the relative error range is $6.9 \% \leq \varepsilon_{S} \leq 19.6 \%$ within usual damping ratio range $0.1 \leq \zeta \leq 0.4$.

Figure 2 shows on conditions of $\zeta=0.4 / 0.3 / 0.2 / 0.1$, the relative error $\varepsilon_{S}$ increases with increasing of $k_{T}$.

\section{Summary}

Based on theoretically modeling, the thesis analyzes and discusses the model errors of momentary impulse action model, which is commonly used in engineering. Specific conclusions are as follows: (1) The measuring principle of momentary impulse action model is based on that the thrust action time is ignored, in specific, less than 1/4 of the pendulum cycle, and the smaller the action time is, and the more accurate are measurements.

(2) The actual thrust always has a certain action time, which deviates from momentary impulse action model, and such deviation is exactly the momentary impulse action model errors. The smaller is the ratio of thrust action time and system cycle, the smaller are the model errors.

(3) Main applications of impulse measurement are: 1) concerning only impulse value, ignoring impulse variation over time, for example, controlling impulse according to the impulse bits; 2) hard to identify impulse variation over time due to too small ratio of thrust action time and system cycle.

\section{References}

[1] Hong Y J, Zhou W J, W G Y, Study on methods for micro-impulse and crucial issues, J. Acta Aeronautica et Astronautica Sinica.34(2013) 2287-2299.

[2] Zhou Weijing, Hong Yanji, Pulsed $\mu$ Newton Thrust Measurement with Torsional Thrust Stand, C. 2nd International Conference on Measurement. Information and Control, Harbin, China. August 16-18 (2013)111-114.

[3] Ye Jifei, etc, Measurement of Laser Ablation Micro Impulse Using the Torsion Pendulum Interferometry,C. The 2nd International Conference on Advanced Measurement and Test, Nanchang 
China. June(2011)1078-1081.

[4] Yan-Ji Hong, Xing Jin et al, Micro-thrust and Micro-impulse Measurement Methods, National defense industry press, Beijing, 2014, pp. 152-155.

[5] Wei-jing Zhou, Yan-ji Hong, Hao Chang, A microNewton thrust stand for average thrust measurement of pulsed microthruster, J. Review of Scientific Instrument. 84 (2013) 115:125. 